\title{
Improving Mouse Models for Dementia. Are All the Effects in Tau Mouse Models Due to Overexpression?
}

\author{
Zelah Joel, ${ }^{1,3}$ Pablo Izquierdo, ${ }^{1}$ Dervis A. Salih, ${ }^{1}$ Jill C. Richardson, ${ }^{2,4}$ \\ Damian M. Cummings, ${ }^{1}$ and Frances A. Edwards ${ }^{1}$ \\ ${ }^{1}$ Department of Neuroscience, Physiology and Pharmacology, University College London, \\ London, WC1E 6BT, United Kingdom \\ ${ }^{2}$ Neurosciences Therapeutic Area, GlaxoSmithKline R\&D, Stevenage, SG1 2NY, United Kingdom \\ Correspondence: f.a.edwards@ucl.ac.uk
}

\begin{abstract}
Mouse models of Alzheimer's disease have commonly used transgenic overexpression of genes involved in production of amyloid $\beta$ (APP and/or PSEN1/2) or Tau (MAPT) with mutations that result in familial forms of dementia. We discuss possible improvements that may create full models while avoiding the problems of overexpression and report synaptic results in APPKI models. We stress use of inappropriate controls without overexpression of the normal human protein and the mismatch between the learning deficits reported in mice with plaques but no tangles and the human condition. We focus on Tau overexpression, including new data that support previous reports of the grossly nonlinear relationship between Tau overexpression and neurofibrillary tangle load, with a twofold increase in Tau protein, resulting in a 100-fold increase in tangle density. These data also support the hypothesis that a high concentration of soluble Tau, in overexpression models, plays an important direct role in neurodegeneration, rather than only via aggregation. Finally, we hypothesize that there is an optimal concentration range over which Tau can bind to microtubules and a threshold beyond which much of the overexpressed protein is unable to bind. The excess thus causes toxicity in ways not necessarily related to the process in human dementias.
\end{abstract}

Alzheimer's disease is the most common form of dementia and, in its later stages, shares common pathologies with frontotemporal dementia and other tauopathies. In all of these dementias, the development of neurodegeneration is closely correlated with the hyperphosphorylation and aggregation of the microtubule-associated protein Tau (MAPT), which typically presents as neurofibrillary tangles or Pick bodies. In the case of Alzheimer's disease, this development is subsequent to a rise in amyloid $\beta(\mathrm{A} \beta)$ and deposition of plaques. Although more than 50 mutations in the MAPT gene have been identified, along with mutations in 21 other genes (for review, see Tacik et al. 2016), the etiology of the majority of tauopathies, including Alzheimer's disease, is sporadic.

Probably the single most important factor that is impeding the progress toward treatments for Alzheimer's disease and other dementias is the lack of good animal models. Although the models that have been used over the last two decades are certainly useful and have given us considerable information, some of the findings may be misleading. It is extremely important to assess carefully what is being modeled and so ask questions that are within the borders of the model used. Even then, of course, all model and experimental tissues (including in vivo and postmortem human tissue) have their limitations and so comparison of different models is often useful and indeed the best approach.
There has been considerable discussion of the possible artifacts due to overexpression of amyloid precursor protein (APP) in mouse models of Alzheimer's disease (for review, see Sasaguri et al. 2017) and some discussion of overexpression of presenilins, which do not only affect APP processing but also have other functions, including modulating the Notch signaling pathway (De Strooper et al. 1999; Selkoe 2001). The problem of overexpression in mice carrying familial Alzheimer's disease mutations (referred to here as "amyloid mice") is now being addressed with the introduction of humanized APP mice expressing combinations of familial mutations (Guo et al. 2013; Saito et al. 2014). With the introduction of recent technologies such as CRISPR, generation of new models has become considerably faster and easier and now these will no doubt be followed by other knock-in and humanized models.

The question of using the correct controls has been a problem across the whole field, with most studies, including our own, using wild-type mice rather than mice bearing the overexpressed human gene without mutations, expressed similarly to the mutated gene in the test animals. Again, this has been a factor of the difficulty and expense of developing such models but is now being addressed. Importantly, the correct controls of humanized APP mice without mutations are now also becoming available for the recently developed knock-in models.

\footnotetext{
${ }^{3}$ Present address: Small Pharma, 3rd Floor, 6-8 Bonhill Street, London, EC2A 4BX, United Kingdom

${ }^{4}$ Present address: MSD, The London Bioscience Innovation Centre (LBIC), 2 Royal College Street, London, NW1 0UT, United Kingdom

(C) 2018 Joel et al. This article is distributed under the terms of the Creative Commons Attribution License, which permits unrestricted reuse and redistribution provided that the original author and source are credited.
} 
In contrast to APP, much less discussion has been raised about the overexpression of Tau, despite transgenic mouse lines overexpressing Tau isoforms or mutant forms of Tau being studied for more than 20 years. A recent review has given valuable insight into different models of Alzheimer's disease with critical suggestions concerning techniques, and this will not be repeated here (Götz et al. 2018). This same review also touched on the importance of overexpression, and it is this point that we would like to expand on here. Recently, several reports have suggested that soluble hyperphosphorylated Tau may be more toxic than tangles themselves (Kopeikina et al. 2012; Cowan and Mudher 2013). It is further suggested that tangles may initially be protective against soluble hyperphosphorylated Tau (d'Orange et al. 2018). Hence, if overexpression of Tau in rodents leads to excess Tau that cannot bind to microtubules, the overexpression itself seems likely to be a major factor in the toxic effects seen, unrelated to whether the Tau carries a mutation that would cause tangles in humans.

Here we briefly discuss amyloid mice, touching on the problems of overexpression and report data on initial comparisons with APPKI models. We also suggest possible approaches that could be used to improve the knock-in models. We then extend the discussion to problems of overexpression of Tau. This is a feature of almost all the available "Tau models" as they are generally transgenic for wild-type or mutated human Tau. We include new data, demonstrating the strongly nonlinear effects that occur when overexpression of Tau exceeds a certain, fairly low, threshold, and we consider to what extent results from such models are relevant to Alzheimer's disease and other dementias.

\section{MOUSE MODELS EXPRESSING FAMILIAL ALZHEIMER'S DISEASE MUTATIONS}

Most of the mouse models that have been used for studying Alzheimer's disease are based on transgenic, or more recently knock-in, expression of human genes carrying mutations that cause the rare familial forms of the disease. These mice generally have raised total $A \beta$ levels and increased ratios of $A \beta_{1-42}$ compared to shorter forms, such as $\mathrm{A} \beta_{1-40}$ and $A \beta_{1-38}$. This results in increasing deposition of plaques over the life of the mouse with a wide range in the rate at which this deposition begins. Interestingly, transgenic models expressing only single mutations in $A P P$ or PSEN1 (as occurs in familial Alzheimer's disease) generally do not feature plaques or not until very late in the life of the mouse. For example, a transgenic mouse that only expresses $A P P$ with the Swedish mutation starts to develop plaques at $\sim 18$ mo of age, although mice with the same mutation combined with a $P S E N 1_{\mathrm{M} 146 \mathrm{~V}}$ mouse start to deposit plaques at $\sim 4$ mo of age (Matarin et al. 2015; Medawar et al. 2018). The PSEN1 mutation alone does not result in plaques within the lifetime of the mouse (Matarin et al. 2015; Medawar et al. 2018). Consequently, to see plaques more rapidly, many models overexpress $A P P$ and either PSEN1 or PSEN2, with both transgenes harboring familial mutations.

\section{Overexpression of $A P P$ and/or PSEN1/2}

The problem of overexpression of $A P P$ and $P S E N 1 / 2$ has been widely recognized. APP is cleaved by a variety of both canonical and noncanonical secretases and caspases (Andrew et al. 2016; Müller et al. 2017), which produce not only $\mathrm{A} \beta$, but a range of other active peptides. These peptides have been shown to exert effects on synaptic transmission and plasticity, and it is clear that the deposition of $\mathrm{A} \beta$ plaques is due to familial mutations carried by the mice that alter the production of $A \beta$ from APP via $\beta$ - and $\gamma$-secretases. However, other effects have been questioned because of the potential role of overexpression. This subject has been recently thoroughly reviewed (Sasaguri et al. 2017). Moreover, this is not a new subject and the definite advantages but also limitations of purely $\mathrm{A} \beta$ pathway models have been discussed for more than a decade (e.g., Spires and Hyman 2005; Radde et al. 2008). We will not repeat this here but rather briefly discuss some initial data comparing the recently developed knock-in mice from RIKEN (Saito et al. 2014) to previous data from overexpression models.

\section{Synaptic Transmission and Plasticity Could Be Particularly Vulnerable to Overexpression Artifacts}

Briefly, although the effect of the mutations on pathology have not been questioned, the effects on synaptic transmission, both early, as pathology is first developing (Cummings et al. 2015), and at later stages (Busche and Konnerth 2015; Medawar et al. 2018), could be highly influenced by overexpression of other APP metabolites. The earliest effect of rising $A \beta$ is an increase in glutamate release probability. Increased release probability occurs even when $A \beta$ concentrations are still very low and with no detectable plaque deposition, and with $\mathrm{A} \beta_{1-40}$ dominating over other forms. This reflects the putative physiological effect of $A \beta$, as shown in wild-type rats (Abramov et al. 2009), and, perhaps not surprisingly, we find that this effect also occurs in APPKI mice (APP ${ }^{\mathrm{NL}-\mathrm{F} / \mathrm{NL}-\mathrm{F}}$ or $A P P^{\text {NL-G-F/NL-G-F }}$ ) before plaques are deposited (Fig. 1). Thus, at least these early changes are probably not artifacts of overexpression. We are in the process of establishing how other changes develop in the knock-in mice with the initial data suggesting that indeed some of the synaptic effects are not recapitulated or at least not until much later in the development of pathology.

Others, however, have already reported additional differences between the knock-in and transgenic amyloid models. For example, endoplasmic reticular stress reported in transgenic mice is not seen in APP ${ }^{\text {NL-G-F/NL-G-F }}$ mice (Hashimoto et al. 2018). Endoplasmic stress results in increased cytoplasmic calcium concentrations, and, consistent with this notion, the substantial accumulation of $\mathrm{A} \beta_{1-42}$ in $\mathrm{APP}^{\mathrm{NL}-\mathrm{F} / \mathrm{NL}-\mathrm{F}}$ mice does not result in the intraneuronal calcium-dependent conversion of p35 to p25 by calpain (Saito et al. 2016).

An alternative approach to humanizing mouse $A P P$ using knock-in approaches has been to use adeno-associated virus-based transfer of human APP and PSEN1 to the 
A

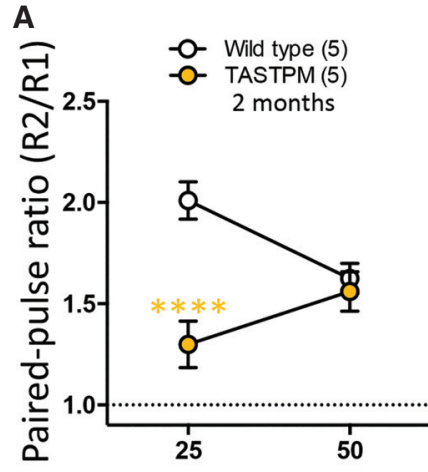

B

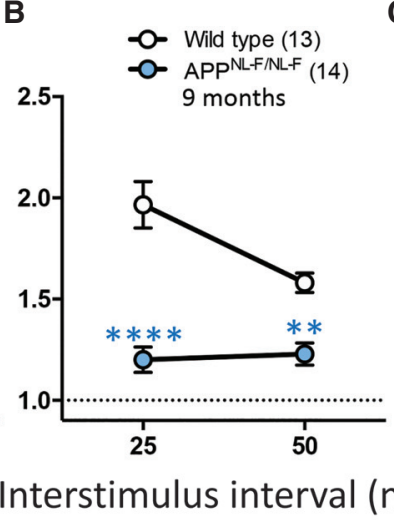

C

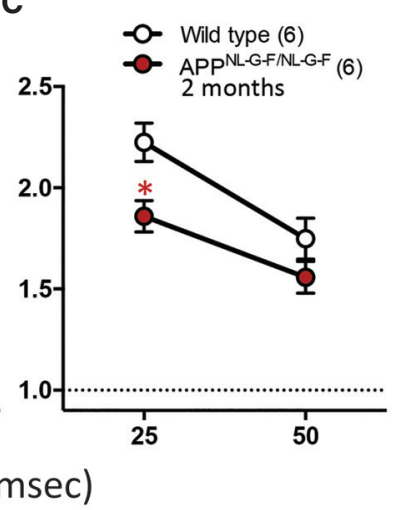

Figure 1. Paired-pulse ratios in transgenic and knock-in APP mice before plaque deposition. Patch clamp recordings of CA1 cells were made to record responses to paired stimulation of CA3 axons. Paired-pulse ratio is inversely proportional to glutamate release probability. (A) TASTPM (transgenic $A P P_{\mathrm{Swe}} / P S E N 1_{\mathrm{M} 146 \mathrm{~V}}$ ) mice at 2 mo of age (plaques from $4 \mathrm{mo}$ ). Data replotted from Cummings et al. $(2015)$ in which detailed methods can be found. $(B) \mathrm{APP}^{\mathrm{NL}-\mathrm{F} / \mathrm{NL}-\mathrm{F}}$ mice at 9 mo of age ( plaques from $\left.10 \mathrm{mo}\right) .(C) \mathrm{APP}^{\mathrm{NL}-\mathrm{G}-\mathrm{F} / \mathrm{NL}-\mathrm{G}-\mathrm{F}} \mathrm{mice}^{-}$ at 2 mo of age (plaques from $4 \mathrm{mo}$ ). Sample sizes are indicated in parentheses. Sidak post hoc tests are indicated following a significant genotype $\times$ interstimulus interval interaction (two-way ANOVA). $(*) P<0.05,(* *) P<0.01,(* * * *) P<0.0001$.

mouse hippocampus, resulting in expression levels similar to that seen in human brain (Audrain et al. 2016). In these mice, $A \beta$ accumulated and resulted in hyperphosphorylation of murine Tau. Although plaques were not detected, synaptic changes were identified at 3 mo postinjection.

It would appear, therefore, that $A \beta$ does in fact have direct effects on synaptic function, independent of other APP metabolites that may be artifactually increased by using overexpression models.

\section{The Problem of Behavioral Deficits; a Mismatch of Model and Experimental Aims}

Behavioral outcomes in Alzheimer's mouse models are a particular problem. Many studies have attempted to define cognitive deficits as an end point in amyloid models and consider the reversal of cognitive deficits to be an important signifier of potentially therapeutically relevant modification of disease progression (Webster et al. 2014; Jankowsky and Zheng 2017).

However, in man, the development of sufficient cognitive deficits to result in diagnosis of the disease does not occur without the development of a substantial tangle load and neurodegeneration (Ridha et al. 2006; Brier et al. 2016). These are the very features that do not occur in such amyloid mice, unless mutations and overexpression of Tau are also included in the model. Hence, the fact that most mouse models with familial mutations in $A P P$ (and/or $P S E N 1 / 2)$ do not go on to develop severe behavioral deficits makes them a good model of Alzheimer's disease, at the stage before when it can presently be diagnosed. The advent of severe cognitive deficits in such models is possibly an artifact of transgenic overexpression or due to use of different promoters, resulting in nonphysiological expression patterns. Hence, the aim of finding and reversing cognitive deficits as a relevant measure in models which do not show Tau pathology and substantial neurodegeneration is a clear mismatch with the stage of the model. This is not to say that there may not be behavioral changes associated with the synaptic effects and subtle network changes caused by the buildup of soluble $A \beta$ and plaques, but that these behaviors are more likely to be reflected in anxiety-related changes, locomotor activity, aggression, and possibly alteration in more natural behaviors. Recent development of in-cage automated monitoring, allowing close observation of normal behaviors, rather than trained cognitive tasks, may lead to a better insight of more relevant behavioral changes (Bains et al. 2018). Such behaviors include nesting, stereotyped behaviors, interaction between mice, sleep patterns, and other activities, particularly during the night when mice are naturally most active.

\section{TAU MODELS; THE PROBLEM OF OVEREXPRESSION OF TAU}

In human tauopathies, the pathological changes in Tau and neurodegeneration are not generally related to changes in the level of Tau protein expression per neuron but rather changes in the sequence of Tau or to imbalances of the different isoforms of Tau or other unknown factors. For example, progressive supranuclear palsy can be caused by variants of the MAPT gene that result in relative accumulation of four-repeat Tau compared to three-repeat Tau (Im et al. 2015). Interestingly, some forms of frontotemporal dementia feature a decrease in soluble Tau protein without the development of neurofibrillary tangles (Zhukareva et al. 2003), suggesting that there is a window of concentration of soluble Tau required for proper function. In Alzheimer's disease, there is no evidence for mutations in Tau or in altered levels of Tau. However, almost all the mouse models of tauopathy, including some for Alzheimer's disease, are created by transgenic expression of human Tau with mutations related to frontotemporal dementia with parkinsonism or other forms of tauopathy. Effects of these mutations are certainly relevant at least to the diseases in which they occur but the effects of overexpression are probably not. 
The question arises: What happens when you overexpress Tau? As recently reviewed (Götz et al. 2018), the problem of overexpression of Tau in transgenic mice was recognized more than a decade ago. In mice overexpressing different fragments of full-length human Tau, even without mutations (Götz et al. 1995; Duff et al. 2000; Andorfer et al. 2003), or indeed overexpressing mouse Tau (Adams et al. 2009), neurofibrillary tangle-like inclusions eventually occur as the expression levels increase and animals age.

Tau is a microtubule-binding protein that is thought to stabilize (Gong and Iqbal 2008) or at least promote assembly of microtubules (Qiang et al. 2018). Tau has also been implicated in the transport of proteins along the axon by competing with binding of kinesin and dynein to the microtubules (Dixit et al. 2008). Tau has many sites of phosphorylation, which relate to normal physiological function. Depending on its phosphorylation state, Tau binds and unbinds from the microtubules, possibly in relation to the transport of proteins up and down the axon (Sengupta et al. 1998). Tau mutations such as P301S or P301L that lead to frontotemporal dementia bind less well to microtubules than wild-type Tau, resulting in increased phosphorylation and loss of function.

We know little about the equilibrium between the number of microtubules and the amount of Tau that can bind to them. Presumably there is an optimal level of Tau protein to allow the correct balance of binding. Excess Tau will be more likely to be unbound and thus more likely to become phosphorylated, resulting in a feed-forward loop inhibiting its ability to bind. Hence, overexpression of Tau may undermine the functional balance between Tau and the microtubules and so tend to allow hyperphosphorylation and permanent dissociation of Tau, resulting in pathology. However, whether phosphorylation of Tau is important for seeding tangle formation is not clear (Goedert et al. 2017). Although, in humans, aggregated Tau is phosphorylated, in vitro experiments suggest that hyperphosphorylation does not make Tau any more prone to aggregation. It is possible that conformational changes lead to hyperphos- phorylation rather than the reverse (Falcon et al. 2015) and likely that other factors are important in aggregation.

It has been suggested that soluble hyperphosphorylated Tau, rather than the Tau tangles themselves, may be the primary factor in cell death (Fox et al. 2011; Gendreau and Hall 2013). Overexpression could also play a role here in mouse models but not in Alzheimer's disease or most other tauopathies, in which there is little evidence for increased levels of Tau protein (Pooler et al. 2014). The presence of "ghost" tangles suggests that at least some neurons with tangles die but cell death exceeds tangle number (SpiresJones and Hyman 2014). However, this could be due to microglia playing a role in removal of extracellular Tau "ghosts" as they can clearly phagocytose phosphorylated Tau in mice with neurofibrillary tangles caused by overexpression of Tau $\mathrm{P}_{\mathrm{P} 301 \mathrm{~L}}$ (Fig. 2). As soluble unbound Tau levels and tangles are likely to be closely correlated, this does not discount the possibility that both soluble hyperphosphorylated Tau and neurofibrillary tangles are toxic via different mechanisms.

\section{Effects of Different Transgene Copy Numbers; a Threshold Level for Toxicity}

In a recent study, we have compared two lines of Tau mice developed by GlaxoSmithKline. The model was originally developed as a single line "TauD35" on a C57BL/6J background, via conventional pronuclear injection techniques. The transgene construct contained the 0N4R isoform of Tau encompassing the P301L mutation, under the CaMK2a promoter (Fig. 3). CaMK2 $\alpha$ has a similar expression distribution within the brain to that of Tau. It is expressed from birth in mice but dramatic increases in CaMK $2 \alpha$ levels are evident by postnatal day 8 , by which time expression within all hippocampal subregions and other forebrain regions appears comparable to that observed in adult mice (Parsley et al. 2007). Such expression minimizes transgene effects on embryonic development and restricts expression to the forebrain.
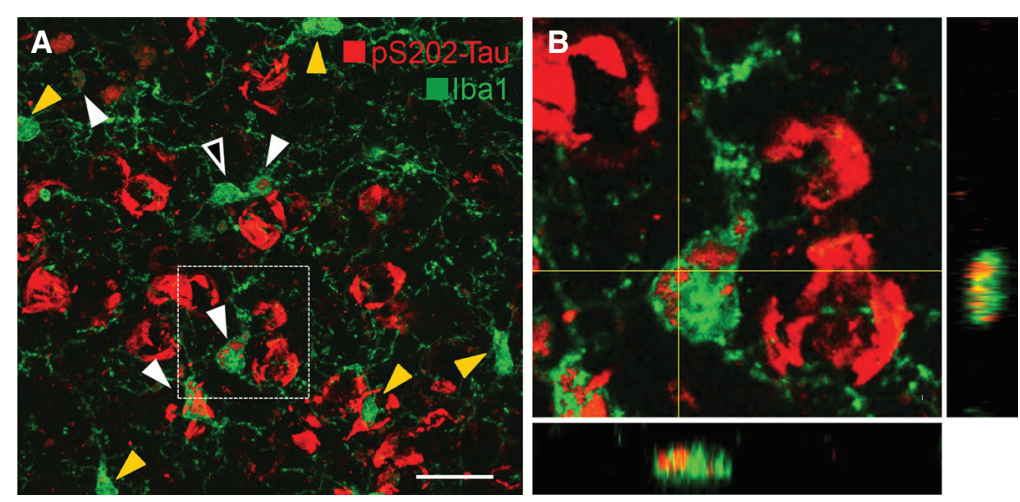

Figure 2. Microglia phagocytose phosphorylated Tau. $(A)$ Representative 2D maximum intensity projection of a $z$-series confocal scan of the cell body layer of the CA1 region of a 24-mo-old lowTAU TauD35 mouse. Microglia are in green (Iba1) and Tau phosphorylated at residue S202 (CP13) are in red. Solid white arrowheads indicate microglia that have phagocytosed pS202-Tau. Solid yellow arrowheads indicate microglia in which very low CP13 positive signal was detected. Open arrowhead indicates a microglia that lacked CP13 signal. Scale bar, $30 \mu \mathrm{m}$. (B) Zoom of indicated area in $A$. Projections in $y z$ and $x z$ are shown at the levels of the crosshairs, demonstrating the internalization of pS202-Tau within the microglia. 
A

B

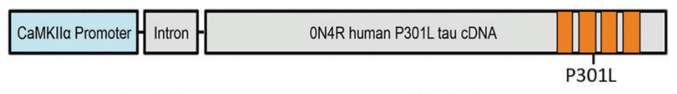

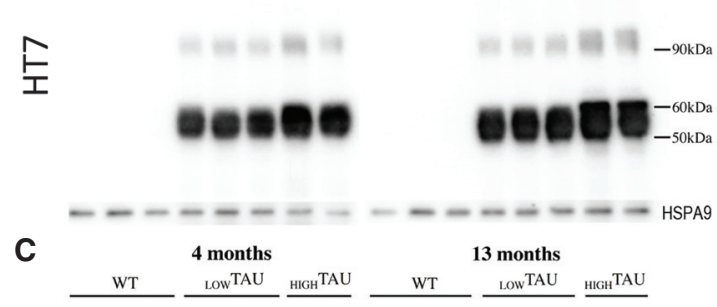

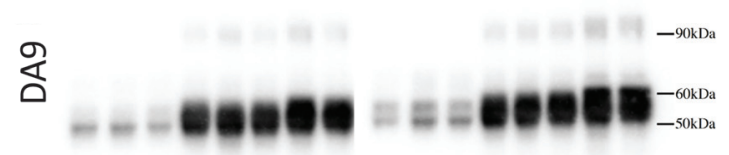

D

$-------------\cdots=$ HSPA9

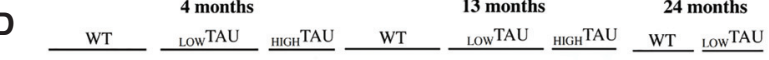
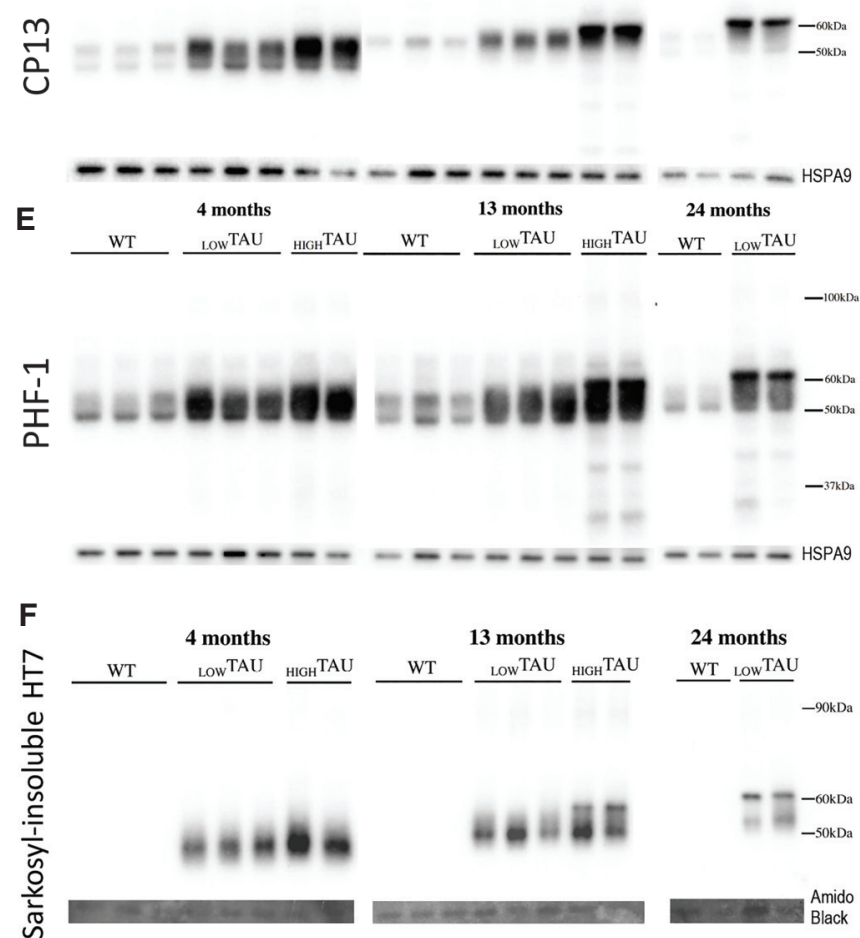
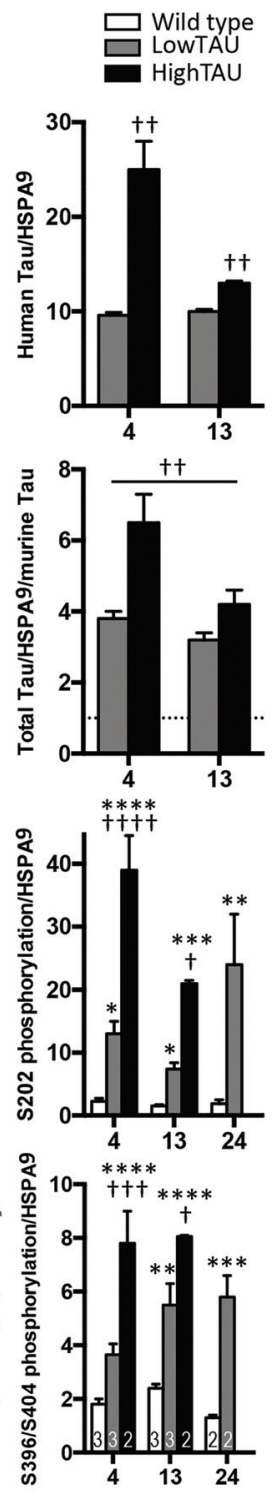

Age (months)

Figure 3. Tau protein expression and phosphorylation in TauD35 mice. (A) Schematic showing TauD35 transgene construct. $(B-F)$ Western blots were performed using antibodies specific to various Tau epitopes and quantified by normalizing intensities to HSPA9. (B) The human Tau-specific antibody HT7 demonstrates the TauD35 transgene is translated into protein. Two-way ANOVA genotype $\times$ age interaction, $P<0.002$. $(C)$ The pan-Tau antibody DA9 was used to examine total Tau (endogenous mouse and transgenic human). Values are normalized to wild-type Tau levels. Note the different sized bands evident in wild type. Two-way ANOVA, main effects of genotype (†) $P<0.003$ and age $P<0.01$; age $\times$ genotype interaction $P=0.06$; Sidak post hoc tests at 4 mo: $P<0.01,13$ mo: $P$ $>0.2$ ). (D) Levels of pS202-Tau (CP13). Age $\times$ genotype interaction: $P<0.0001$. (E) Levels of pS396/S404-Tau (PHF-1). Two-way ANOVA age $\times$ genotype interaction: $P<0.05$. $(F)$ Human Tau (HT7) within the sarkosyl-insoluble fraction. Note, amido black (total protein) was used as a loading control; however, the low levels of protein in this fraction make quantification impractical. Sample sizes (animals) for all antibodies are indicated by the numbers within columns in $E$. Sidak post hoc tests with respect to wild type are indicated by (*) $P<0.05,(* *) P<0.01,(* * *) P<0.001,(* * * *) P<0.0001$; and with respect to lowTAU by $(\dagger) P<0.05,(\dagger \dagger) P<0.01$,

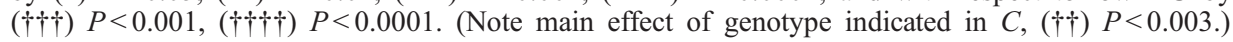


Despite coming from one initial founder line, when we estimated the transgene copy number, we found that there were in fact two separate lines; a lowTAU line with about five transgene copies and a highTAU line with around twice as many copies. We thus assessed the transgene copy number in all mice by designing a qPCR experiment using uniquely designed TaqMan primers/probe for the CaMK $2 a$ promoter sequence and comparing this to the endogenous level of two copies of CaMK2a.

Although we could not analyze the original founder and $F_{1}$ offspring, the most likely explanation for the emergence of two lines with different copy numbers was that the original line had two separate integration sites, with a higher and lower copy number, and that, as hemizygous offspring were bred, these two transgene integrants rapidly segregated. Hence, although the development of the two lines was identical (using the same method of generation, the same promoter, and coming from the same source), they may also have different integration sites in addition to their different copy numbers.

Increase in Tau Protein Levels as Expected from Doubling the Gene Copy Number. Using western blots, the relative levels of human Tau, compared to a housekeeping protein, were estimated in the highTAU and lowTAU mice at 4 mo of age (Fig. 3). Total Tau was also measured and compared to the endogenous mouse Tau. Tau protein levels were close to what would be expected from the estimated copy numbers, with the highTAU mice showing approximately double the level of human Tau protein as the lowTAU mice. When total Tau was measured compared to mouse Tau, the lowTAU mice showed approximately four times more Tau than the wild-type mice, whereas the highTAU showed approximately 6.5 times the mouse Tau level. As the transgenic mice are hemizygous for human Tau but retain two copies of the mouse Tau, this is consistent with expected expression levels, considering the approximate copy number estimates.

Interestingly, at $13 \mathrm{mo}$ of age, the differences in soluble Tau expression between the highTAU and lowTAU mice was almost lost. This is likely to be due to Tau forming into insoluble tangles, which are not detected by the western blot as it only measures soluble Tau levels.

\footnotetext{
Doubling Human Mutated Tau Levels Had a Dramatic Effect on Occurrence of Tau Tangles and Neurodegeneration. We went on to analyze different phosphorylation sites and the appearance of tangle-like Tau deposits in the cell bodies of the hippocampal pyramidal neurons (Fig. 4). At 13 mo of age, the primary signal for phosphorylated Tau was within the axons, for example, between the CA3 and CA1 regions of the hippocampus. A few tangle-like structures could be detected in the cell bodies of CA1 cells. However, the highTAU mice at the same age showed weaker axonal staining but a very heavy load of deposited Tau in the cell bodies, particularly of CA1 cells but also in the dentate and CA3 (Fig. 4C,D). The Tau tangle load in the CA1 region of the highTAU mice was around 100-fold the level seen in the lowTAU
}

mice, despite the protein having the same mutation and the levels being only around double.

Note that, as mentioned above, the soluble Tau levels at this age were almost equal but more Tau was detectable in the sarkosyl-insoluble fraction from the highTAU mice than the lowTAU mice. We interpret this to mean that much of the Tau in the highTAU mouse was not detected on the western blot because it had dissociated from microtubules and deposited in the cell bodies as neurofibrillary tangles. The remaining level of soluble Tau was not substantially different from the level at 4 mo, suggesting that this is close to a threshold for the concentration of overexpressed Tau that can remain soluble and in equilibrium, binding to microtubules without deposition (Fig. 3).

Hence, the relationship between protein level and tangle formation is far from linear. This was also true for neurodegeneration. Once the mice had aged to $\sim 18 \mathrm{mo}$, the highTAU mice developed a distinctive neurodegenerative phenotype, presenting as immobility, piloerection, and a hunched posture. It was therefore necessary to cull the mice at this point and their brains were analyzed and compared to age-matched wild-type littermates. The brains of highTAU mice at this stage showed distinct neurodegeneration with a smaller brain size and large ventricles (Fig. 5). Cell counts revealed that there was substantial loss of around two-thirds of the neurons in the CA1 region compared to wild-type mice. This neurodegeneration started by 13 mo and progressed rapidly in highTAU mice. In lowTAU mice, the tangle load eventually reached a similar level to the highTAU mice at $13 \mathrm{mo}$, but there was no detectable neurodegeneration. It was also notable that the CA1 region was considerably more prone to neurodegeneration than the $\mathrm{CA} 3$ region (Fig. 5B,C).

This supports previous suggestions that not only is the relationship between mutated Tau protein level to tangles nonlinear, but that the neurodegeneration is not entirely dependent on tangle load, depending, at least partly, on the history of exposure to soluble Tau. It is interesting to note that rather than age increasing the effect of a particular tangle load, the effect on neuronal loss in the 24-mo-old lowTAU mice was so little as to be undetectable, unlike the significant loss of neurons in the 13-mo highTAU mice, despite a similar tangle count.

\section{CONCLUSION}

The above data strongly support previous suggestions (as reviewed in Götz et al. 2018) that overexpression is a major factor in the degree to which mouse models show neurofibrillary tangles. In this experiment, the comparison is between two lines of otherwise identical mice, expressing the same mutation hemizygously, under the same conditions on the same promoter, with the only difference being copy number (and possibly transgene insertion site). Serendipitously, the copy number difference seems to span a threshold at which tangles begin early and aggressively in the highTAU mice and hardly at all until almost a year later in the lowTAU mice. Moreover, this aggressive onset of tangles in the highTAU mice, or the 
Ai

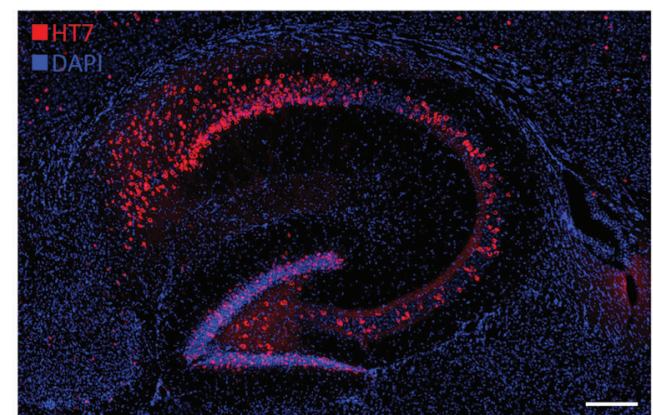

Aii

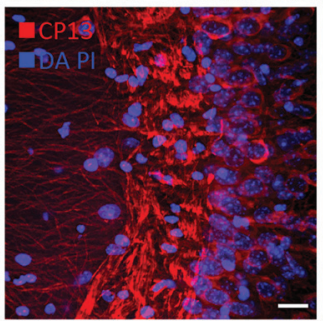

4 months

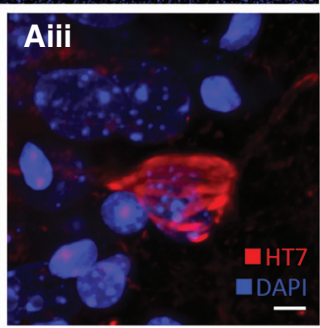

13 months

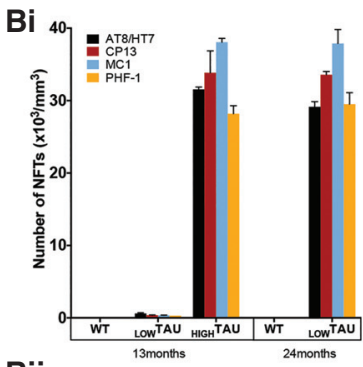

Bii

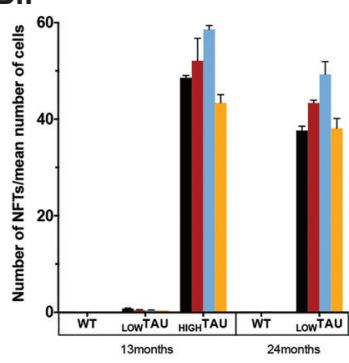

24 months
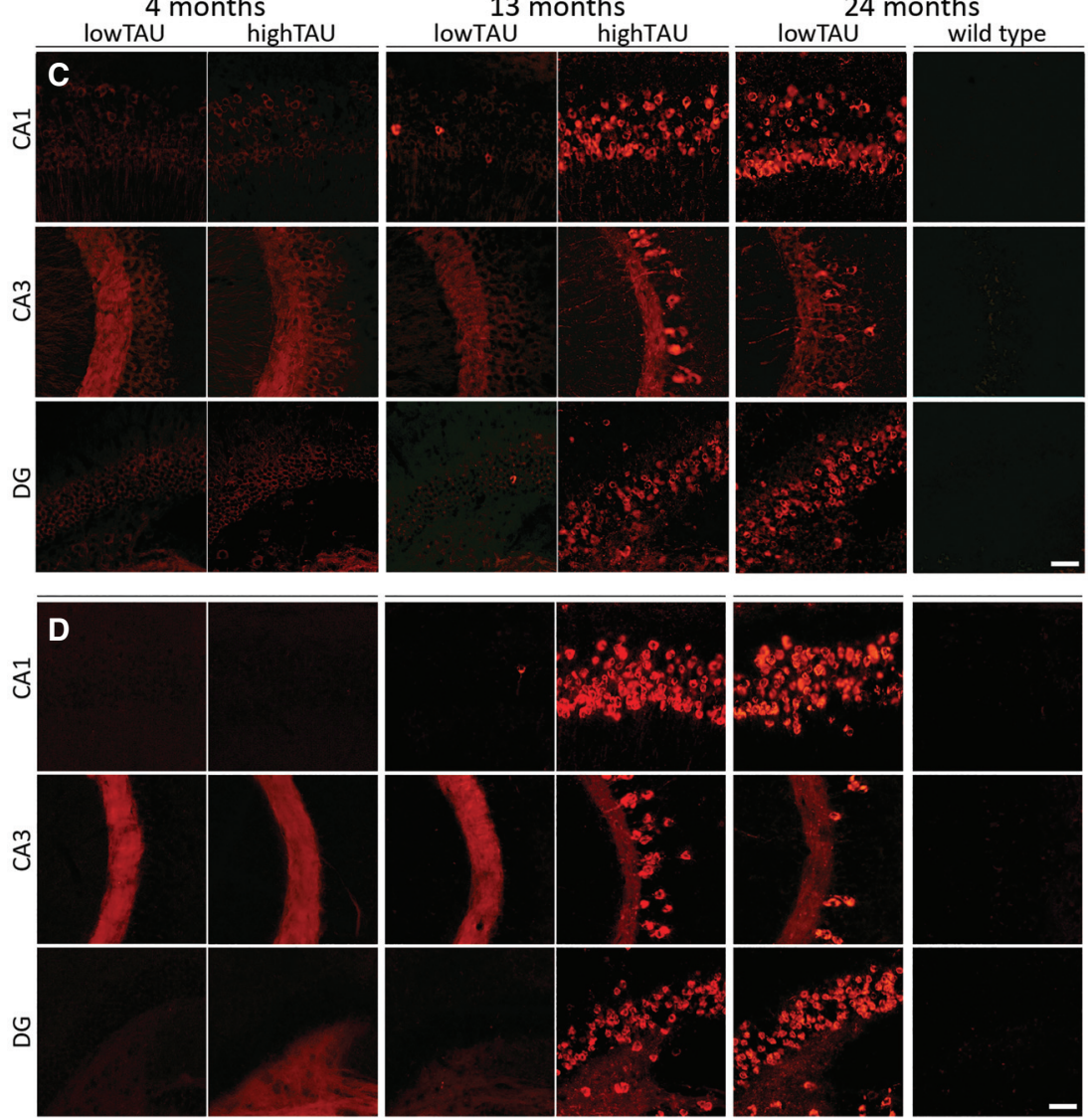

Figure 4. Neurofibrillary tangles in highTAU mice. ( $\mathrm{A}$ i) Overall image of transverse section of hippocampus from a 13-mo-old highTAU mouse, double stained using HT7 (human Tau, red) and DAPI (nuclei, blue). (Composite image using a 20× objective on an EVOS FL Auto Cell Imaging System.) Scale bar, $200 \mu \mathrm{m}$. (Aii) CA3 region of a 4-mo-old highTAU mouse double stained using CP13 (pS202Tau) and DAPI. Note diffuse Tau staining within all cellular compartments, in particular the somatodendritic compartment. Scale bar, $20 \mu \mathrm{m}$. (Aiii) A single CA3 neuron from Ai showing twisted Tau filaments filling the soma. Scale bar, $5 \mu \mathrm{m}$. (Images Aii and Aiii obtained using a Zeiss LSM 510 confocal microscope with $60 \times$ oil immersion objective.) (Bi) Quantification of Tau immunofluorescence detected with either AT8/HT7 double staining, CP13, MC1, or PHF-1 in CA1. (Bii) Data from Bi normalized to the number of neurons counted (Fig. 5C). (C) Example of immunofluorescence images obtained from CA1, CA3, and dentate gyrus at the ages and genotypes indicated. Sections were incubated with the primary antibody CP13, which detects pS202-Tau. Scale bar, $25 \mu \mathrm{m}$. (D) Example of immunofluorescence images obtained from CA1, CA3, and dentate at the ages and genotypes indicated. Note the strong staining of axons that decreases as Tau tangle density increases. Sections were incubated with the primary antibody MC1, which detects misfolded Tau. Scale bar, $25 \mu \mathrm{m}$. 


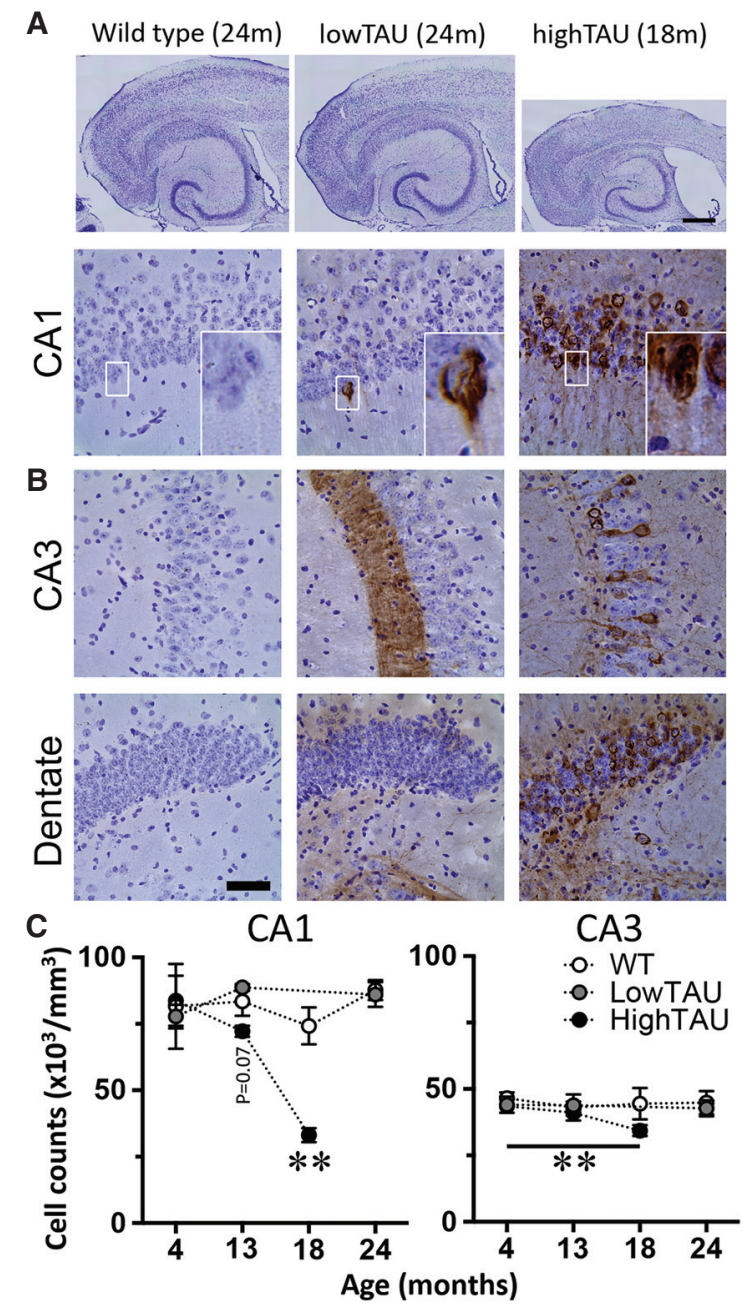

Figure 5. Neuronal cell loss in TauD35 mice. (A) Cresyl violetstained sections of brain from 18-mo-old or 24-mo-old animals, as indicated, showing hippocampus, dorsal ventricle, and surrounding cortex. Note the distinct shrinkage of total brain tissue, an enlargement of the ventricle and thinning of the cortex in the highTAU mice. Scale bar, $500 \mu \mathrm{m}$. (B) Sections of primary neuronal cell body layers of CA1, CA3, and dentate gyrus stained in brown for AT8 (Tau) and counterstained with cresyl violet. Insets show mislocation of Tau to the somatic and dendritic compartments and neurofibrillary tangle-like structures within the soma in Tau mice. Scale bar, $50 \mu \mathrm{m}$. (C) Neuronal cell counts in CA1 and CA3 indicate substantial cell loss. (CA1) Two-way ANOVA age $\times$ genotype interaction for wild-type versus highTAU mice, $P<0.03$. Sidak post hoc test $(* *) P<0.01$. No significant main effects or interaction for wild type versus lowTAU. (Separate two-way ANOVAs run for lowTAU vs. wild type and highTAU vs. wild type to accommodate missing age groups.) (CA3) Main effect of genotype, $P<0.01$. (NB) HighTAU mice are euthanized at $16.5-19$ mo of age due to the advanced neurodegenerative phenotype; $n=2-4$ per genotype per age.

greater concentration of soluble Tau causing them, is much more damaging than the lower level of mutated human Tau in lowTAU mice from which tangles gradually develop, even when the tangle load in the lowTAU mice eventually reaches the same level as had occurred, nearly a year earlier in the highTAU mice.

\section{Hypothesis}

A particular type of axon may have a certain range of concentrations of Tau that provides the optimal binding to allow its physiological functions in relation to microtubules. In mice overexpressing Tau, the concentration of Tau is higher. Up to a certain concentration the Tau present will continue to contribute functionally. However, with more competition between molecules, each molecule would be bound less of the time than would be the case at a lower concentration. This would result in a gradual tendency for molecules that are more often unbound to become hyperphosphorylated, leading to toxicity. However, past a certain concentration threshold, many molecules would bind very rarely, or not at all, increasing their vulnerability to rapid hyperphosphorylation and formation of Tau tangles and/or to causing toxicity directly.

In the case of a mouse also expressing physiological levels of wild-type murine Tau, the murine Tau may bind more efficiently to the microtubules than the human Tau, especially if the latter carries a mutation, and this will keep the microtubules healthy for longer but will further decrease the chance of transgenic Tau binding. In the absence of murine Tau, the human Tau may never fully fulfill the physiological functions of the murine Tau on murine microtubules and so, although competition for binding to the microtubules would be less, the effect of overexpression may occur at even lower levels of Tau. It is interesting to note that in a mouse in which the P301L mutation was knocked in (and so was not overexpressed), there was no evidence of tangle formation (Gilley et al. 2016). Moreover, when a doxycycline-regulatable P301L-overexpressing mouse was studied, turning off expression of the transgene halted or even reversed phenotype progression (Spires et al. 2006). Furthermore, immunization with antibodies to Tau have been successful in ameliorating the effects of overexpression in several mouse models which may simply reflect loss of toxic overexpression rather than a clinically relevant effect (for review, see Novak et al. 2018).

Overexpression of normal human Tau shows some pathology but not to the extent seen in the data presented here or in other mutant Tau models. Nevertheless, the question arises as to how much of a role the overexpression takes in the formation of Tau tangles and in neurodegeneration; this also applies to the many other conclusions from Tau studies that are largely dependent on overexpression models, such as the prion-like propagation of Tau through anatomically connected regions (for review, see Goedert et al. 2017). Is the release of transgenic Tau dependent on the concentration of phosphorylated Tau present in the neuron? There is at least some evidence that aggregated Tau can propagate through the network in wild-type mice without overexpression if extracts from the brains of people who have died with advanced-stage tauopathies are injected into the mouse brain (Clavaguera et al. 2013). Although this effect is much stronger if the extracts are injected into mice overexpressing Tau, so that overexpression again clearly has a role, it nevertheless shows that propagation is possible in wild-type mice and moreover the aggregates distal from the 
injection site contain mouse Tau. Further questions also arise considering the types of Tau that make up the different tangles and how these different forms of Tau would behave under normal and overexpression conditions (Fitzpatrick et al. 2017). Certainly, in Alzheimer's disease, in which Tau shows neither overexpression nor mutations, and in which the tangles contain both three- and four-repeat Tau, the dysfunctional Tau may behave very differently from the mutant Tau or even normal human Tau that is overexpressed beyond certain thresholds in mouse models and its pathological behavior may depend on different factors.

\section{How Do We Improve the Mouse Models for Alzheimer's Disease and Other Tauopathies?}

Certainly, knock-in of proteins is generally more likely to avoid artifacts due to overexpression or expression at nonphysiological times or places. However, this tends not to produce full models of the various dementias, and in particular, without overexpression, it seems to be difficult to get a mouse to form Tau tangles, even with mutations. Mouse Tau may be more resistant to toxic change, and so replacing mouse with human Tau may be effective, although this may also cause artifacts if human Tau is incompatible with mouse microtubules. Rats may be an interesting alternative to mice, as rat Tau is more similar to human Tau and rats live longer (Hanes et al. 2009; Cohen et al. 2013). Knock-in of the appropriate forms of the genes that are known to trigger the diseases ( $A P P$, $P S E N s$, or $M A P T$ ) and adding other risk factors may help to change the course of disease progression. For example, some of the microglial genes such as TREM2 seem to be protective in Alzheimer's disease and when mutated lose their protective function (Ulland et al. 2017; Ulrich et al. 2017). In mice, such genes are strongly expressed in response to plaque development (Matarin et al. 2015; Salih et al. 2018), and this may provide the mouse with protection from the full effects of rising $\mathrm{A} \beta$ (Keren-Shaul et al. 2017). Knocking down or mutating these genes in the mouse or rat, together with other risk factors, may be effective. The approach of using knock-in of familial Alzheimer's genes to supply the trigger for the initial rise of $\mathrm{A} \beta$ and combining this with manipulation of other genetic or environmental risk factors may well increase the chance of exacerbating disease progression. Even if not creating a full model of Alzheimer's disease, this approach will certainly be informative in terms of the role of different pathways in disease progression and the link between $A \beta$ and Tau. The search for additional risk factors that may lead to toxicity due to Tau, without overexpression, is only just beginning.

\section{ACKNOWLEDGMENTS}

APPKI mice were generously provided by Professors Takaomi Saido and Takashi Saito, RIKEN, Japan. The Tau D35 mice were supplied by GlaxoSmithKline. We thank Karina Vitanova for comments on the manuscript and Professor Michel Goedert, University of Cambridge, for very helpful discussion. We also thank Prof. Peter Davies (The Feinstein Institute for Medical Research) for the generous gift of the Tau antibodies. This work was supported by GlaxoSmithKline, EPSRC, the Dementia Research Institute, and the Medical Research Council UK.

\section{REFERENCES}

Abramov E, Dolev I, Fogel H, Ciccotosto GD, Ruff E, Slutsky I. 2009. Amyloid- $\beta$ as a positive endogenous regulator of release probability at hippocampal synapses. Nat Neurosci 12: 1567 1576. doi: $10.1038 / \mathrm{nn} .2433$

Adams SJ, Crook RJ, Deture M, Randle SJ, Innes AE, Yu XZ, Lin WL, Dugger BN, McBride M, Hutton M, et al. 2009. Overexpression of wild-type murine tau results in progressive tauopathy and neurodegeneration. Am J Pathol 175: 15981609. doi:10.2353/ajpath.2009.090462

Andorfer C, Kress Y, Espinoza M, de Silva R, Tucker KL, Barde YA, Duff K, Davies P. 2003. Hyperphosphorylation and aggregation of tau in mice expressing normal human tau isoforms. J Neurochem 86: 582-590. doi:10.1046/j.1471-4159. 2003.01879.x

Andrew RJ, Kellett KA, Thinakaran G, Hooper NM. 2016. A Greek tragedy: the growing complexity of Alzheimer amyloid precursor protein proteolysis. J Biol Chem 291: 19235-19244. doi:10.1074/jbc.R116.746032

Audrain M, Fol R, Dutar P, Potier B, Billard JM, Flament J, Alves S, Burlot MA, Dufayet-Chaffaud G, Bemelmans AP, et al. 2016. Alzheimer's disease-like APP processing in wild-type mice identifies synaptic defects as initial steps of disease progression. Mol Neurodegener 11: 5. doi:10.1186/s13024-0160070-y

Bains RS, Wells S, Sillito RR, Armstrong JD, Cater HL, Banks G, Nolan PM. 2018. Assessing mouse behaviour throughout the light/dark cycle using automated in-cage analysis tools. $J$ Neurosci Methods 300: 37-47. doi:10.1016/j.jneumeth.2017 .04 .014

Brier MR, Gordon B, Friedrichsen K, McCarthy J, Stern A, Christensen J, Owen C, Aldea P, Su Y, Hassenstab J, et al. 2016. Tau and $A \beta$ imaging, CSF measures, and cognition in Alzheimer's disease. Sci Transl Med 8: 338ra366. doi: 10.1126/scitranslmed.aaf2362

Busche MA, Konnerth A. 2015. Neuronal hyperactivity-a key defect in Alzheimer's disease? Bioessays 37: 624-632. doi:10.1002/bies.201500004

Clavaguera F, Akatsu H, Fraser G, Crowther RA, Frank S, Hench J, Probst A, Winkler DT, Reichwald J, Staufenbiel M, et al. 2013. Brain homogenates from human tauopathies induce tau inclusions in mouse brain. Proc Natl Acad Sci 110: 95359540. doi:10.1073/pnas.1301175110

Cohen RM, Rezai-Zadeh K, Weitz TM, Rentsendorj A, Gate D, Spivak I, Bholat Y, Vasilevko V, Glabe CG, Breunig JJ, et al. 2013. A transgenic Alzheimer rat with plaques, tau pathology, behavioral impairment, oligomeric $A \beta$, and frank neuronal loss. J Neurosci 33: 6245-6256. doi:10.1523/JNEUROSCI. 3672-12.2013

Cowan CM, Mudher A. 2013. Are tau aggregates toxic or protective in tauopathies? Front Neurol 4: 114. doi:10.3389/ fneur.2013.00114

Cummings DM, Liu W, Portelius E, Bayram S, Yasvoina M, Ho SH, Smits H, Ali SS, Steinberg R, Pegasiou CM, et al. 2015. First effects of rising amyloid- $\beta$ in transgenic mouse brain: synaptic transmission and gene expression. Brain 138: 1992-2004. doi:10.1093/brain/awv127

d'Orange M, Aurégan G, Cheramy D, Gaudin-Guérif M, Lieger S, Guillermier M, Stimmer L, Joséphine C, Hérard AS, Gaillard MC, et al. 2018. Potentiating tangle formation reduces acute toxicity of soluble tau species in the rat. Brain 141: 535-549. doi:10.1093/brain/awx342

De Strooper B, Annaert W, Cupers P, Saftig P, Craessaerts K, Mumm JS, Schroeter EH, Schrijvers V, Wolfe MS, Ray WJ, 
et al. 1999. A presenilin-1-dependent $\gamma$-secretase-like protease mediates release of Notch intracellular domain. Nature 398: 518-522. doi:10.1038/19083

Dixit R, Ross JL, Goldman YE, Holzbaur EL. 2008. Differential regulation of dynein and kinesin motor proteins by tau. Science 319: 1086-1089. doi:10.1126/science.1152993

Duff K, Knight H, Refolo LM, Sanders S, Yu X, Picciano M, Malester B, Hutton M, Adamson J, Goedert M, et al. 2000. Characterization of pathology in transgenic mice over-expressing human genomic and cDNA tau transgenes. Neurobiol Dis 7: 87-98. doi:10.1006/nbdi.1999.0279

Falcon B, Cavallini A, Angers R, Glover S, Murray TK, Barnham L, Jackson S, O’Neill MJ, Isaacs AM, Hutton ML, et al. 2015. Conformation determines the seeding potencies of native and recombinant Tau aggregates. J Biol Chem 290: 1049-1065. doi:10.1074/jbc.M114.589309

Fitzpatrick AWP, Falcon B, He S, Murzin AG, Murshudov G, Garringer $\mathrm{HJ}$, Crowther RA, Ghetti B, Goedert M, Scheres SHW. 2017. Cryo-EM structures of tau filaments from Alzheimer's disease. Nature 547: 185-190. doi:10.1038/nature23002

Fox LM, William CM, Adamowicz DH, Pitstick R, Carlson GA, Spires-Jones TL, Hyman BT. 2011. Soluble tau species, not neurofibrillary aggregates, disrupt neural system integration in a tau transgenic model. J Neuropathol Exp Neurol 70: 588595. doi:10.1097/NEN.0b013e318220a658

Gendreau KL, Hall GF. 2013. Tangles, toxicity, and tau secretion in $\mathrm{AD}$ - new approaches to a vexing problem. Front Neurol 4: 160. doi:10.3389/fneur.2013.00160

Gilley J, Ando K, Seereeram A, Rodríguez-Martín T, Pooler AM, Sturdee L, Anderton BH, Brion JP, Hanger DP, Coleman MP. 2016. Mislocalization of neuronal tau in the absence of tangle pathology in phosphomutant tau knockin mice. Neurobiol Aging 39: 1-18. doi:10.1016/j.neurobiolaging.2015.11.028

Goedert M, Eisenberg DS, Crowther RA. 2017. Propagation of Tau aggregates and neurodegeneration. Annu Rev Neurosci 40: 189-210. doi:10.1146/annurev-neuro-072116-031153

Gong CX, Iqbal K. 2008. Hyperphosphorylation of microtubuleassociated protein tau: a promising therapeutic target for Alzheimer disease. Curr Med Chem 15: 2321-2328. doi:10.2174/ 092986708785909111

Götz J, Probst A, Spillantini MG, Schäfer T, Jakes R, Bürki K, Goedert M. 1995. Somatodendritic localization and hyperphosphorylation of tau protein in transgenic mice expressing the longest human brain tau isoform. EMBOJ 14: 1304-1313. doi:10.1002/j.1460-2075.1995.tb07116.x

Götz J, Bodea LG, Goedert M. 2018. Rodent models for Alzheimer disease. Nat Rev Neurosci 19: 583-598. doi:10.1038/ s41583-018-0054-8

Guo Q, Li H, Cole AL, Hur JY, Li Y, Zheng H. 2013. Modeling Alzheimer's disease in mouse without mutant protein overexpression: cooperative and independent effects of $A \beta$ and tau. PLoS One 8: e80706. doi:10.1371/journal.pone. 0080706

Hanes J, Zilka N, Bartkova M, Caletkova M, Dobrota D, Novak M. 2009. Rat tau proteome consists of six tau isoforms: implication for animal models of human tauopathies. J Neurochem 108: 1167-1176. doi:10.1111/j.1471-4159.2009.05869.x

Hashimoto S, Ishii A, Kamano N, Watamura N, Saito T, Oshima T, Yokosuka M, Saido TC. 2018. Endoplasmic reticulum stress responses in mouse models of Alzheimer disease: overexpression paradigm versus knock-in paradigm. $J$ Biol Chem 293: 3118-3125. doi:10.1074/jbc.M117.811315

Im SY, Kim YE, Kim YJ. 2015. Genetics of progressive supranuclear palsy. J Mov Disord 8: 122-129. doi:10.14802/jmd. 15033

Jankowsky JL, Zheng H. 2017. Practical considerations for choosing a mouse model of Alzheimer's disease. Mol Neurodegener 12: 89. doi:10.1186/s13024-017-0231-7

Keren-Shaul H, Spinrad A, Weiner A, Matcovitch-Natan O, DvirSzternfeld R, Ulland TK, David E, Baruch K, Lara-Astaiso D, Toth B, et al. 2017. A unique microglia type associated with restricting development of Alzheimer's disease. Cell 169: 1276-1290.e17. doi:10.1016/j.cell.2017.05.018
Kopeikina KJ, Hyman BT, Spires-Jones TL. 2012. Soluble forms of tau are toxic in Alzheimer's disease. Transl Neurosci 3: 223-233. doi:10.2478/s13380-012-0032-y

Matarin M, Salih DA, Yasvoina M, Cummings DM, Guelfi S, Liu W, Nahaboo Solim MA, Moens TG, Paublete RM, Ali SS, et al. 2015. A genome-wide gene-expression analysis and database in transgenic mice during development of amyloid or tau pathology. Cell Rep 10: 633-644. doi:10.1016/j.celrep .2014.12.041

Medawar E, Benway TA, Liu W, Hanan TA, Haslehurst P, James OT, Yap K, Muessig L, Moroni F, Nahaboo Solim MA, et al. 2018. Effects of rising amyloid $\beta$ on hippocampal synaptic transmission, microglial response and cognition in $A P P_{\text {Swe }} /$ ${ }_{P S E N 1} 1_{\mathrm{M} 146 \mathrm{~V}}$ transgenic mice. EBioMedicine 39: 422-435. doi:10.1016/j.ebiom.2018.12.006

Müller UC, Deller T, Korte M. 2017. Not just amyloid: physiological functions of the amyloid precursor protein family. Nat Rev Neurosci 18: 281-298. doi:10.1038/nrn.2017.29

Novak P, Kontsekova E, Zilka N, Novak M. 2018. Ten years of tau-targeted immunotherapy: the path walked and the roads ahead. Front Neurosci 12: 798. doi:10.3389/fnins.2018.00798

Parsley SL, Pilgram SM, Soto F, Giese KP, Edwards FA. 2007. Enriching the environment of $\alpha \mathrm{CaMKIIT}^{286 \mathrm{~A}}$ mutant mice reveals that LTD occurs in memory processing but must be subsequently reversed by LTP. Learn Mem 14: 75-83. doi: 10.1101/lm.356607

Pooler AM, Noble W, Hanger DP. 2014. A role for tau at the synapse in Alzheimer's disease pathogenesis. Neuropharmacology 76: 1-8. doi:10.1016/j.neuropharm.2013.09.018

Qiang L, Sun X, Austin TO, Muralidharan H, Jean DC, Liu M, Yu W, Baas PW. 2018. Tau does not stabilize axonal microtubules but rather enables them to have long labile domains. Curr Biol 28: 2181-2189.e4. doi:10.1016/j.cub.2018.05.045

Radde R, Duma C, Goedert M, Jucker M. 2008. The value of incomplete mouse models of Alzheimer's disease. Eur J Nucl Med Mol Imaging 35: S70-S74. doi:10.1007/s00259-0070704-y

Ridha BH, Barnes J, Bartlett JW, Godbolt A, Pepple T, Rossor MN, Fox NC. 2006. Tracking atrophy progression in familial Alzheimer's disease: a serial MRI study. Lancet Neurol 5: 828-834. doi:10.1016/S1474-4422(06)70550-6

Saito T, Matsuba Y, Mihira N, Takano J, Nilsson P, Itohara S, Iwata N, Saido TC. 2014. Single App knock-in mouse models of Alzheimer's disease. Nat Neurosci 17: 661-663. doi: 10.1038/nn.3697

Saito T, Matsuba Y, Yamazaki N, Hashimoto S, Saido TC. 2016. Calpain activation in Alzheimer's model mice is an artifact of APP and presenilin overexpression. J Neurosci 36: 99339936. doi:10.1523/JNEUROSCI.1907-16.2016

Salih DA, Bayram S, Guelfi MS, Reynolds RH, Shoai M, Ryten M, Brenton J, Zhang D, Matarin M, Botia J, et al. 2018. Genetic variability in response to $A \beta$ deposition influences Alzheimer's risk. bioRxiv doi:10.1101/437657

Sasaguri H, Nilsson P, Hashimoto S, Nagata K, Saito T, De Strooper B, Hardy J, Vassar R, Winblad B, Saido TC. 2017. APP mouse models for Alzheimer's disease preclinical studies. EMBO J 36: 2473-2487. doi:10.15252/embj.201797397

Selkoe DJ. 2001. Presenilin, Notch, and the genesis and treatment of Alzheimer's disease. Proc Natl Acad Sci 98: 1103911041. doi:10.1073/pnas.211352598

Sengupta A, Kabat J, Novak M, Wu Q, Grundke-Iqbal I, Iqbal K. 1998. Phosphorylation of tau at both Thr 231 and Ser 262 is required for maximal inhibition of its binding to microtubules. Arch Biochem Biophys 357: 299-309. doi:10.1006/abbi.1998 .0813

Spires-Jones TL, Hyman BT. 2014. The intersection of amyloid $\beta$ and tau at synapses in Alzheimer's disease. Neuron 82: 756771. doi:10.1016/j.neuron.2014.05.004

Spires TL, Hyman BT. 2005. Transgenic models of Alzheimer's disease: learning from animals. NeuroRx 2: 423-437. doi: 10.1602/neurorx.2.3.423

Spires TL, Orne JD, SantaCruz K, Pitstick R, Carlson GA, Ashe KH, Hyman BT. 2006. Region-specific dissociation of neuro- 
nal loss and neurofibrillary pathology in a mouse model of tauopathy. Am J Pathol 168: 1598-1607. doi:10.2353/ajpath .2006 .050840

Tacik P, Sanchez-Contreras M, Rademakers R, Dickson DW, Wszolek ZK. 2016. Genetic disorders with tau pathology: a review of the literature and report of two patients with tauopathy and positive family histories. Neurodegener Dis 16: 1221. doi: $10.1159 / 000440840$

Ulland TK, Song WM, Huang SC, Ulrich JD, Sergushichev A, Beatty WL, Loboda AA, Zhou Y, Cairns NJ, Kambal A, et al 2017. TREM2 maintains microglial metabolic fitness in Alzheimer's disease. Cell 170: 649-663.e13. doi:10.1016/j.cell. 2017.07.023
Ulrich JD, Ulland TK, Colonna M, Holtzman DM. 2017. Elucidating the role of TREM2 in Alzheimer's disease. Neuron 94: 237-248. doi:10.1016/j.neuron.2017.02.042

Webster SJ, Bachstetter AD, Nelson PT, Schmitt FA, Van Eldik LJ. 2014. Using mice to model Alzheimer's dementia: an overview of the clinical disease and the preclinical behavioral changes in 10 mouse models. Front Genet 5: 88. doi:10.3389/fgene.2014.00088 Zhukareva V, Sundarraj S, Mann D, Sjogren M, Blenow K, Clark CM, McKeel DW, Goate A, Lippa CF, Vonsattel JP, et al. 2003. Selective reduction of soluble tau proteins in sporadic and familial frontotemporal dementias: an international follow-up study. Acta Neuropathol 105: 469-476. doi:10.1007/s00401002-0668-8 


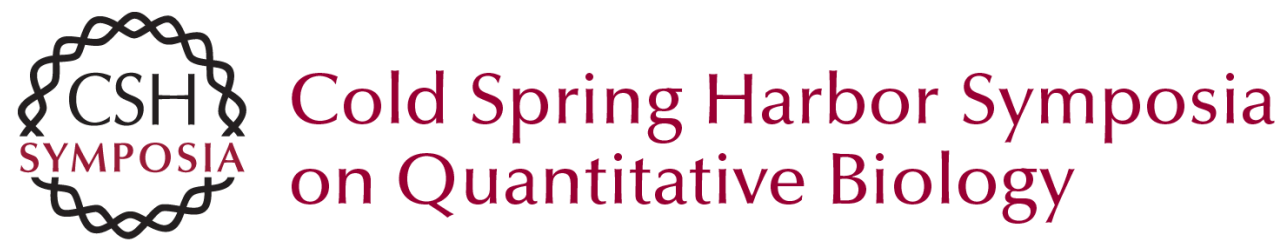

\section{Improving Mouse Models for Dementia. Are All the Effects in Tau Mouse Models Due to Overexpression?}

Zelah Joel, Pablo Izquierdo, Dervis A. Salih, et al.

Cold Spring Harb Symp Quant Biol 2018 83: 151-161 originally published online February 11, 2019

Access the most recent version at doi:10.1101/sqb.2018.83.037531

References This article cites 55 articles, 11 of which can be accessed free at: http://symposium.cshlp.org/content/83/151.full.html\#ref-list-1

Creative This article is distributed under the terms of the

Commons http://creativecommons.org/licenses/by/4.0/, which permits unrestricted

License reuse and redistribution provided that the original author and source are credited.

Email Alerting Receive free email alerts when new articles cite this article - sign up in Service the box at the top right corner of the article or click here. 